\title{
Indication and Timing of Thyroid Surgery for Patients with Hereditary Medullary Thyroid Cancer Syndromes
}

\author{
Jennifer B. Ogilvie, MD, and Electron Kebebew, MD, San Francisco, California
}

\author{
Key Words \\ Familial medullary thyroid cancer, MEN 2A, MEN 2B, FMTC, RET, \\ prophylactic thyroidectomy
}

\begin{abstract}
Hereditary medullary thyroid cancer syndromes comprise familial medullary thyroid cancer (FMTC) and multiple endocrine neoplasia types $2 \mathrm{~A}$ and $2 \mathrm{~B}$. Hereditary medullary thyroid cancers have an autosomal dominant pattern of inheritance and are caused by activating germline point mutations in the RET proto-oncogene. Evaluation of the onset, extent, and progression of hereditary medullary thyroid cancer associated with specific RET mutations has enabled clinicians to treat patients based on the level of risk associated with their specific mutation. Children identified by RET screening to be at risk for the development of medullary thyroid cancer can be treated with prophylactic thyroidectomy before developing the disease. This review covers the diagnosis, evaluation, timing of surgical management, and optimal follow-up of patients with hereditary medullary thyroid cancer syndromes. (JNCCN 2006;4:139-147)
\end{abstract}

Hereditary medullary thyroid cancer (MTC) accounts for $25 \%$ of MTC, or $1 \%$ to $3 \%$ of all thyroid cancers. ${ }^{1} \mathrm{MTC}$ is a neuroendocrine tumor of the calcitonin-producing parafollicular $\mathrm{C}$ cells derived from the embryonic neural crest. Hereditary MTC is one of the best understood inherited neuroendocrine tumors because the 3 patterns of

From the University of California, San Francisco, Department of Surgery, Endocrine Surgery and Oncology Program, San Francisco, California. Submitted July 22, 2005; accepted for publication October 5, 2005.

Dr. Kebebew is supported in part by the Harold Amos Faculty Development Program of the Robert Wood Johnson Foundation, Hellman Family Grant, and the University of California Cancer Research Committee (EK). The authors have no financial interest, arrangement, or affiliation with the manufacturers of any products discussed in the article or their competitors.

Correspondence: Electron Kebebew MD, University of California, San Francisco, Department of Surgery, Endocrine Surgery and Oncology Program, 1600 Divisadero Street, Box 1674, San Francisco, CA 94143.

E-mail: kebebewe@surgery.ucsf.edu. autosomal dominant inheritance result from germline point mutations in the RET proto-oncogene. The RET (REarranged during Transfection) proto-oncogene was identified in $1985 .{ }^{2}$ It encodes a receptor tyrosine kinase and is located on chromosome 10q11.2. In 1993, RET was identified as the susceptibility gene for familial MTC (FMTC) and multiple endocrine neoplasia type 2A (MEN 2A) ${ }^{3,4}$ In 1994, mutations in the RET proto-oncogene were also found to be responsible for MEN type 2B (MEN 2B)..$^{5}$ RET screening is now used to identify family members at risk for developing MTC and to help determine the optimal treatment approach. The genotype and phenotype features of the 3 hereditary MTC syndromes are summarized in Table 1.

\section{Diagnosis and Workup of Familial Medullary Thyroid Cancer}

Index cases of hereditary MTC commonly present like sporadic cases, with a palpable thyroid mass or enlarged cervical lymph nodes and sometimes with pheochromocytoma or hyperparathyroidism. The highest density of parafollicular C cells is in the posterior upper third of each thyroid lobe. Most medullary thyroid cancers occur in this region. Associated symptoms of hoarseness, dysphagia, or shortness of breath are seen in up to $15 \%$ of patients and often indicate aggressive tumors that have invaded the recurrent laryngeal nerve, trachea, or esophagus. ${ }^{6}$ Any patient presenting with these symptoms should undergo direct laryngoscopy to assess the function of the recurrent laryngeal nerve. Additional imaging with computed tomography (CT) or magnetic resonance imaging (MRI) should also be considered to define the extent of tumor invasion.

Regional cervical lymph node involvement is seen in up to $80 \%$ of patients with primary tumor size $1 \mathrm{~cm}$ or 
Ogilvie and Kebebew

\begin{tabular}{|c|c|c|c|c|c|c|}
\hline \multirow[b]{2}{*}{ Syndrome } & \multirow{2}{*}{$\begin{array}{l}\text { Incidence } \\
\text { of MTC }\end{array}$} & \multirow{2}{*}{$\begin{array}{l}\text { Youngest Age } \\
\text { at Presentation } \\
\text { of MTC }\end{array}$} & \multirow[b]{2}{*}{ Associated Tumors } & \multicolumn{3}{|c|}{ RET Codon Mutations } \\
\hline & & & & Level 1 & Level II & Level III \\
\hline FMTC & $100 \%$ & $<6 y$ & None & $\begin{array}{l}768790791 \\
804891\end{array}$ & $\begin{array}{l}609611618 \\
620630634\end{array}$ & \\
\hline MEN 2A & $95 \%$ & $<1 \mathrm{y}$ & $\begin{array}{l}\text { Pheochromocytoma, } 40 \% \\
\text { Hyperparathyroidism, 20\% } \\
\text { Cutaneous lichen } \\
\text { amyloidosis, 9\% } \\
\text { +/- Hirschsprung's }\end{array}$ & 790791 & $\begin{array}{l}609611618 \\
620634\end{array}$ & \\
\hline MEN 2B & $100 \%$ & $<9$ mo & $\begin{array}{l}\text { Pheochromocytoma, } 70 \% \\
\text { Intestinal/mucosal } \\
\text { ganglioneuromatosis } \\
\text { Marfanoid features }\end{array}$ & & & 883918922 \\
\hline
\end{tabular}

Abbreviations: FMTC, familial medullary thyroid cancer syndrome; MEN 2A, multiple endocrine neoplasia, type 2A; MEN 2B, multiple endocrine neoplasia, type 2B; MTC, medullary thyroid cancer; RET, REarranged during Transfection.

larger and $100 \%$ of patients with tumors that have invaded through the thyroid capsule. ${ }^{7,8}$ Lymph node metastases occur most commonly in the central cervical compartment $(56 \%)$, followed by the ipsilateral (49\%) and contralateral (22\%) cervical compartments. ${ }^{9}$ The central and lateral cervical lymph node compartments are shown in Figure 1. Distant metastases of MTC to the liver, lung, and bone are seen in $12 \%$ to $15 \%^{8}$ of patients and can be associated with flushing and diarrhea from tumor production of calcitonin, calcitonin gene-related peptide, or other vasoactive substances. ${ }^{10}$ Occasionally, patients with MTC present with hypertensive crises or other complications of pheochromocytoma. ${ }^{9}$ Rarely, MTC produces ectopic adrenocorticotropic hormone (ACTH), causing Cushing's syndrome or hyperparathyroidism.

To establish the diagnosis of medullary thyroid cancer in a patient with a neck mass, fine-needle aspiration (FNA) biopsy should be performed. The diagnosis can be confirmed with positive immunohistochemical staining for calcitonin, carcinoembryonic antigen (CEA), and amyloid, and negative staining for thyroglobulin. After the diagnosis of MTC has been established, serum basal or calcium-stimulated calcitonin and CEA levels should be measured. Calcium-stimulated calcitonin has replaced pentagastrin stimulation (no longer available in the United States), and involves the intravenous administration of calcium $(2 \mathrm{mg} / \mathrm{kg})$ over 1 minute, with serum calcitonin levels measured at 2, 5, and 10 minutes after administration. A positive test is defined as calcitonin at least 2 times the upper limit of normal after calcium stimulation. Preoperative calcitonin levels directly correlate with tumor size, and preoperative calcitonin levels less than $50 \mathrm{pg} / \mathrm{mL}$ are associated with postoperative calcitonin normalization in $98 \%$ of patients (compared with only $42 \%$ of patients with preoperative calcitonin greater than $50 \mathrm{pg} / \mathrm{mL}){ }^{11}$ Falsely elevated calcitonin can be caused by autoimmune (Hashimoto's) thyroiditis and mutinodular goiter. ${ }^{12}$ All patients should have genetic testing for germline mutations in the RET proto-oncogene.

The physical examination of patients with MTC should include complete neck ultrasound, including careful examination of the thyroid gland for additional nodules, and examination of all cervical lymph node compartments for enlarged or suspicious lymph nodes. All patients with MTC should also have preoperative

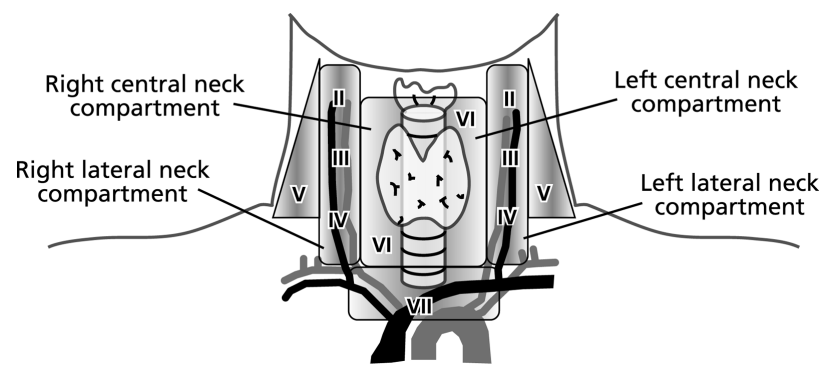

Figure 1 Central and lateral cervical lymph node compartments. Level VI nodes comprise the central compartment and Levels II, III, IV and V comprise the lateral compartment. 
biochemical testing for pheochromocytoma. Approximately $40 \%$ of patients with MEN 2 have a pheochromocytoma. ${ }^{13}$ Pheochromocytomas in MEN 2 are more often bilateral, are confined to the adrenal gland, and have a lower risk of malignancy than sporadic pheochromocytomas. ${ }^{10,14}$ Patients with elevated urinary or serum catecholamine and metabolites should undergo an abdominal CT scan with fine cuts through the adrenal glands to localize a pheochromocytoma. Patients with a pheochromocytoma should be treated with an alpha blocker (phenoxybenzamine, $10 \mathrm{mg} 3$ times a day, titrated up to $40 \mathrm{mg} 3$ times a day) for at least 2 weeks before surgical intervention. Surgery in a patient with an undiagnosed pheochromocytoma without alpha blockade can be fatal. In patients with MTC and a pheochromocytoma, the adrenalectomy should be performed before total thyroidectomy and after appropriate alpha blockade.

A complete family history is essential to identify cases of hereditary MTC syndromes. All patients should be questioned about a family history of thyroid cancer, pheochromocytomas, hyperparathyroidism, or clinical manifestations such as neck mass, hoarseness, hypertension, anxiety, sweating, palpitations, headache, fatigue, weakness, memory loss, depression, musculoskeletal aches and pains, kidney stones, and osteoporosis. Serum calcium should be measured to rule out hyperparathyroidism. Finally, all patients with MTC should undergo germline RET screening, because $6 \%$ to $24 \%$ of patients with apparently sporadic MTC have germline RET mutations. ${ }^{15,16}$ Patients and families with germline RET mutations should undergo appropriate genetic counseling before genetic testing.

\section{RET Screening}

Germline RET proto-oncogene mutations have been identified in exons 10,11, 13, 14, 15, and 16 through direct DNA sequence analysis. The most common mutation is in exon 11, codon 634, which is seen in two thirds of patients with hereditary MTC syndromes (FMTC and MEN 2A). RET screening identifies index cases of hereditary MTC syndromes and can identify which family members are at risk for developing MTC, eliminating the need for yearly routine calcitonin screening. Early identification of patients at risk for MTC using RET screening resulted in a 95\% biochemical cure rate at 6-year follow-up in 1 study, com- pared with a $33 \%$ biochemical cure rate in patients who were diagnosed clinically with hereditary MTC. ${ }^{9}$

Certain RET mutations are associated with an earlier age of onset and more aggressive tumors. This genotype-phenotype relationship should be used to determine the optimal timing of prophylactic thyroidectomy in families with hereditary MTC (Table 1). To date, no false-negative or false-positive RET tests have been reported in family members of a patient with MEN 2 with a detectable RET mutation. An estimated $5 \%$ to $10 \%$ of families with hereditary MTC do not have a described RET mutation and thus may have a false-negative result. These family members should be followed up with yearly basal and calciumstimulated calcitonin levels.

\section{Surgical Treatment of Medullary Thyroid Cancer}

The only effective treatment for MTC is surgical resection. Parafollicular $\mathrm{C}$ cells do not trap iodine like thyroid follicular cells. Therefore, radioiodine ablation is not effective. MTC is generally more aggressive than differentiated thyroid cancers, and local recurrence in the thyroid bed or cervical lymph nodes occurs in more than $50 \%$ of patients after surgical resection of established palpable tumors. Hereditary MTC is almost always multicentric and bilateral, with premalignant diffuse $\mathrm{C}$-cell hyperplasia present even in the absence of ultrasound abnormalities. ${ }^{9,17,18}$ Overall, central neck lymph node metastases occur in at least $50 \%$ of patients with hereditary MTC that present with a palpable mass, with ipsilateral lymph node involvement in $28 \%$ and contralateral lymph node involvement in $19 \% .^{19}$

Patients with a diagnosis of MTC should undergo total thyroidectomy with central cervical lymph node (levels VI, VII) and ipsilateral functional modified radical lymph node (levels II, III, IV, V) resection. If abnormal contralateral lymph nodes are noted using ultrasound or palpation or if bilateral primary tumors are found, a contralateral functional modified radical neck lymph node dissection should also be performed. Total thyroidectomy involves the extracapsular removal of the thyroid lobes, isthmus, and pyramidal lobe, sparing the recurrent laryngeal nerve and parathyroid glands. If the blood supply to the parathyroid glands appears to be compromised or if recurrence in the region of the parathyroid glands is a concern, the 
parathyroid glands may be transplanted into the sternocleidomastoid muscle (sporadic, FMTC, or MEN 2B) or the nondominant forearm brachioradialis muscle (MEN 2A). Complete central neck lymph node dissection includes removal of all lymphatic and fibrofatty tissue between the 2 carotid arteries laterally, and from the hyoid bone superiorly down to the anterior superior mediastinum. A functional modified radical lymph node dissection involves the removal of all lymphatic and fibrofatty tissue along the jugular chain from the angle of the mandible superiorly to the innominate vessels inferiorly and from the anterior border of the trapezius muscle laterally to the anterior scalene muscles. The jugular vein, sternocleidomastoid muscle, and phrenic, spinal accessory, vagus, cervical sensory, and brachial plexus nerves are left intact.

Patients with a germline RET mutation and a thyroid nodule or elevated calcitonin should undergo total thyroidectomy with central neck lymph node resection, plus an ipsilateral lateral function neck dissection if the central lymph nodes are positive for MTC. Patients with a germline RET mutation and no other evidence of MTC should undergo prophylactic total thyroidectomy. The timing of prophylactic thyroidectomy is based on the risk level of each specific RET mutation (Table 1).

Recurrent or residual MTC should be suspected when serum calcitonin is detectable after complete resection. Calcitonin levels greater than $1,000 \mathrm{pg} / \mathrm{mL}$ may indicate distant metastases in the liver or lungs. ${ }^{20}$ The workup of recurrent MTC should start with a review of the initial surgery and pathologic findings to ensure that an appropriate surgical resection was performed. A recent review of over 1,000 patients with MTC in the Surveillance, Epidemiology, and End Results (SEER) database between 1973 and 2000 showed that even over the past 8 years, $15 \%$ of patients had only a subtotal or thyroid lobectomy, and $41 \%$ had no lymph node dissection. ${ }^{21}$ Subsequent evaluation should include complete neck ultrasound, direct laryngoscopy, and chest and abdominal CT. Hepatic metastases of MTC are often too small $(<3$ $\mathrm{mm}$ ) to be visualized with a CT scan, and a laparoscopic examination of the liver may be helpful for adequate staging before a redo neck operation. Nuclear medicine evaluation of MTC includes positronemission tomography (PET) scan, octreotide scan and bone scans. ${ }^{22,23}$ If all evaluations are negative, selec- tive venous catheterization for stimulated calcitonin measurement to localize the recurrence may be helpful. ${ }^{24}$ Observation alone is appropriate for patients with elevated calcitonin levels and no detectable disease.

Metastatic MTC to the liver with symptomatic diarrhea and flushing can be treated with resection or radiofrequency ablation. ${ }^{9}$ Lung metastases of MTC may be resectable thoracoscopically or through open thoracotomy. In general, isolated metastases of MTC should be resected whenever possible.

\section{Prognosis}

Many clinicopathologic tumor staging systems have been proposed to predict prognosis and risk of recurrence for MTC. The American Joint Committee on Cancer (AJCC) and Union International Contre le Cancer (UICC) pTNM, the European Organization for Research and Treatment of Cancer (EORTC), the National Thyroid Cancer Treatment Cooperative Study (NTCTCS), and SEER staging systems for MTC are shown in Table 2. The overall 10-year survival for patients with MTC is approximately $75 \%$ (range, $61 \%-88 \%) .{ }^{25,26}$ In addition to stage, multivariate analysis has identified age at diagnosis as one of the most important independent predictors of survival, with 10 -year survival rates of $75 \%$ in patients younger than 40 years and only $50 \%$ in patients over age 40 at diagnosis. ${ }^{8,27}$ All the staging systems have been shown to be accurate predictors of survival. Poor prognostic pathologic features include strong CEA or galectin-3 staining, weak calcitonin staining, and cellular heterogeneity. ${ }^{28-30}$

\section{Adjuvant Treatment for Medullary Thyroid Cancer}

\section{Chemotherapy}

Despite meticulous surgical resection, at least $50 \%$ of patients presenting with clinically evident MTC will have local recurrence or distant metastases. Many patients survive for years with detectable levels of calcitonin or metastatic disease. ${ }^{31}$ Adjuvant chemotherapy with cytotoxic single agents such as doxorubicin produces a partial, short-lived response in less than $40 \%{ }^{32,33}$ Small trials using combination chemotherapy (dacarbazine, 5-FU, epirubicin, vincristine, cyclophosphamide, and cisplatin) have also produced only 
Thyroid Surgery for Medullary Thyroid Cancer Syndromes

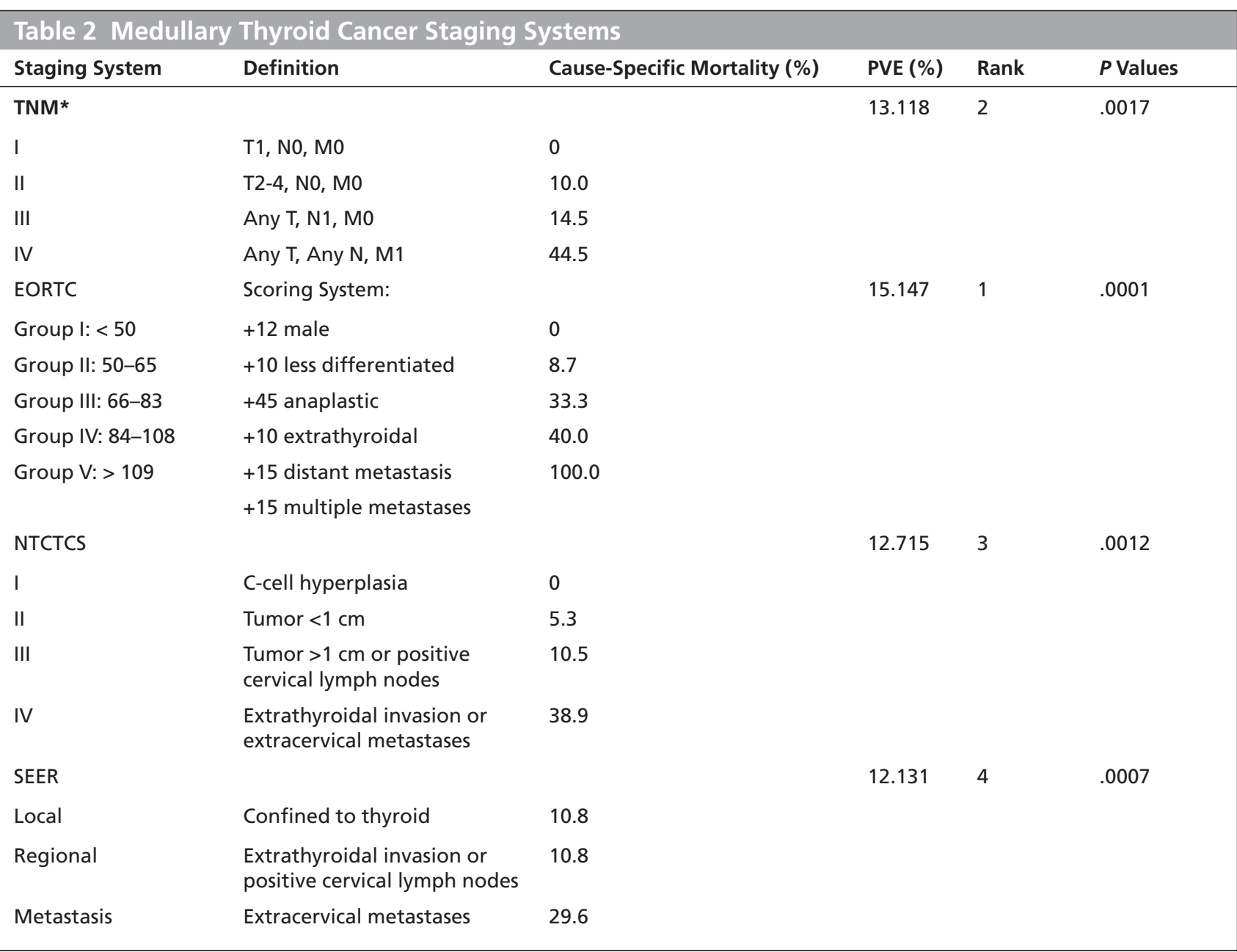

Abbreviations: EORTC, European Organization for Research and Treatment of Cancer; NTCTCS, National Thyroid Cancer Treatment Cooperative Study; SEER, Surveillance, Epidemiology and End Results; PVE, proportion of variance explained.

*Tumor size: $\mathrm{T} 1 \leq 1 \mathrm{~cm} ; \mathrm{T} 2>1 \mathrm{~cm}$ but $<4 \mathrm{~cm}$; T3 $\geq 4 \mathrm{~cm}$; T4 extrathyroidal invasion.

Lymph node: $\mathrm{NO}=$ no regional lymph node metastases; $\mathrm{N} 1$ = positive cervical lymph node metastases. Metastasis:

$\mathrm{M} 0=$ no distant metastases; $\mathrm{M} 1=$ positive distant metastases.

partial responses or short-term disease stabilization. ${ }^{34-39}$ A phase II clinical trial of irinotecan in patients with metastatic MTC is ongoing. A preclinical study in nude mice of combrestatin A-4 phosphate (CA4P) with doxorubicin showed significant antitumor activity against human MTC in a xenograft model. ${ }^{40}$

Somatostatin analogues, including octreotide and lanreotide, used alone or with interferon $\alpha 2 \mathrm{~b}$ improve symptoms of flushing and diarrhea in over $60 \%$ of patients, and lower calcitonin and CEA levels in a few patients, but have not been shown to reduce tumor volume. ${ }^{41,42}$ Arylidene-2-indolinone (RPI-1), a small molecule inhibitor, reverses the constitutive tyrosine phosphorylation caused by the RET 634 mutation in MEN 2A and is a novel approach to the treatment of hereditary MTCs. ${ }^{43}$ Other studies using tyrosine kinase inhibitors in vitro have shown promising results, ${ }^{44,45}$ and phase II clinical trials are underway.

Gene therapy using dominant negative RET mutants or RNA interference to block RET expression is another potential treatment approach being investigated. ${ }^{46,47}$ A phase I trial using ${ }^{131}$ I-labeled anti-CEA monoclonal antibodies (131I-MN-14) with autologous hematopoietic stem cell rescue in 12 patients reported 1 partial response. ${ }^{48}$ Another study used vaccination with calcitonin-pulsed dendritic cells as immunotherapy in 7 patients with 1 partial response. ${ }^{49}$ A different immunotherapeutic approach would be to develop monoclonal antibodies against the 
Ogilvie and Kebebew

ectodomain of RET, analogous to the use of trastuzumab against ErbB2 in the treatment of breast cancer. ${ }^{47}$

\section{Radiation}

No survival benefit has been documented with external beam radiation in the treatment of MTC, ${ }^{50}$ although more recent retrospective studies have suggested an improvement in local control, especially in patients with locally invasive tumors or positive tumor margins. One small cohort study of patients with residual microscopic MTC in the neck showed that locoregional control at 10 years was $86 \%$ in the group treated with postoperative external beam radiation versus $52 \%$ in the group that did not receive postoperative radiation. ${ }^{51}$ An important consideration in the use of external beam radiation for the treatment of MTC is that radiationinduced fibrosis and vasculitis may make repeat neck surgery much more difficult. Complications of radiation include myelitis $(0.5 \%)$, esophagitis, laryngitis, and late esophageal stricture (5\%)..$^{52}$ External beam radiation therapy may be useful in patients with very aggressive or unresectable tumors.

\section{Familial Medullary Thyroid Cancer}

\section{Presentation}

Familial MTC is characterized as a variant of MEN 2A with no other manifestations other than MTC. The criteria for FMTC are strict and require more than 10 patients with identifiable RET mutations or MTC in the kindred, with multiple carriers or affected patients over age 50 years. ${ }^{53}$ Patients with FMTC usually present by age 30 , although some patients present before the age of 5 .

\section{Screening}

The disease is associated with moderate-risk level II RET mutations (codons 609, 611, 618, 620, 634) that can cause MTC before age 5 and lower-risk level I RET mutations (codons 768, 790, 791, 804, 891) that have more variable penetrance and a later age of onset (usually not before age 10; Table 1). Researchers have estimated that up to $5 \%$ of kindreds with FMTC do not yet have identified RET mutations and thus may have negative RET screening results in the known exons.

\section{Prophylactic Surgical Intervention}

Patients with FMTC should undergo prophylactic thyroidectomy by age 5 to 10 , depending on the risk of their individual RET mutations (before 5 years for level II RET mutations and before 10 years for level I RET mutations). A central neck lymph node dissection should be performed for concomitant thyroid nodules, elevated calcitonin levels, or abnormal lymph nodes on ultrasound or palpation.

\section{Multiple Endocrine Neoplasia Type 2A}

\section{Presentation}

Multiple endocrine neoplasia 2A is associated with MTC in over $95 \%$ of patients, pheochromocytoma in $40 \%$, and multiple gland parathyroid hyperplasia in 20\%. Cutaneous lichen amyloidosis may be present in some patients (9\%) with MEN 2A and is characterized by a pigmented, scaly rash in the interscapular area or on the extensor surfaces of the extremities. ${ }^{54}$ Cutaneous lichen amyloidosis also occurs sporadically and as a familial disease in patients without MEN 2A or identified RET mutations. Patients with MEN 2A usually develop MTC by the third decade of life, although patients developing MTC before the age of 2 have been reported.

\section{Screening}

Over $95 \%$ of MEN 2A index patients have identified RET mutations on germline screening. ${ }^{52}$ MEN 2A RET mutations are usually located in the extracellular cysteine residues on exons 10 and 11, most commonly at codon 634. Patients with higher-risk level II mutations $(609,611,618,620,634)$ should undergo total prophylactic thyroidectomy before 5 years of age. The appropriate time for thyroidectomy in patients with low-risk level I mutations $(790,791)$ is more controversial because the penetrance is highly variable, but these patients should probably also undergo thyroidectomy before 5 years of age (Table 1 ).

\section{Prophylactic Surgical Intervention}

Patients with MEN 2A MTC or a thyroid nodule and elevated calcium-stimulated calcitonin levels should undergo total thyroidectomy, central neck lymph node dissection, and ipsilateral lateral functional neck lymph node dissection. Patients with palpable or ultrasound evidence of contralateral disease should also undergo contralateral lymph node dissection. Prophylactic thyroidectomy for patients with MEN 2A and no evidence of MTC should be performed before age 5, especially for patients with higher-risk level II mutations. 
Thyroid Surgery for Medullary Thyroid Cancer Syndromes

Intraoperative management of the parathyroid glands is controversial, because $20 \%$ of patients with MEN 2A have multiple gland parathyroid hyperplasia. Some surgeons advocate routine total parathyroidectomy with autotransplantation into the nondominant forearm brachioradialis muscle. ${ }^{55}$ Advocates of this approach suggest that it permits a more complete lymph node dissection and avoids the risk of repeat neck dissection for hyperparathyroidism in the future. Other surgeons advocate leaving the parathyroid glands in situ with their vascular pedicles intact, marked with a surgical clip for future identification. ${ }^{12,56}$ If the parathyroid's vascular supply appears to be compromised, the gland should be confirmed by frozen section, then autotransplanted into the forearm.

\section{Multiple Endocrine Neoplasia Type 2B}

\section{Presentation}

Medullary thyroid cancer associated with MEN 2B presents within the first 2 decades of life and tends to be the most aggressive form of hereditary MTC. Researchers have reported metastatic MTC in patients as young as 1 year of age..$^{77,58}$ Other features of MEN 2B include pheochromocytoma, marfanoid body habitus, mucosal neuromas of the lips and oral cavity, intestinal ganglioneuromas, and occasionally chronic constipation associated with colonic motility disorders and megacolon.

\section{Screening}

RET proto-oncogene mutations in codon 833 or 918 cause MEN 2B..$^{59}$ This high-risk level III mutation may cause MTC as early as 6 months of age and is present in about $25 \%$ of patients with sporadic MTC as a somatic mutation (Table 1).

\section{Prophylactic Surgical Intervention}

Because of the aggressive nature of MTC associated with MEN 2B, all patients with identified RET mutations should undergo prophylactic thyroidectomy before 1 year of age, ideally in the first month of life. Prophylactic central neck lymph node dissection should be performed if suspicious lymph nodes are seen on physical examination or ultrasound, or a thyroid nodule or elevated calcitonin level is detected preoperatively. ${ }^{8}$ Central neck lymph node dissection in children should always be performed by skilled endocrine surgeons who can identify and preserve or transplant the parathyroid glands.

\section{Recommendations}

Appropriate RET screening, diagnosis, and timing and approach of surgical treatment of hereditary MTC is critical in the management of this often challenging disease. Complete surgical resection, including total thyroidectomy, central neck lymph node dissection, and, when indicated, lateral ipsilateral or bilateral functional modified radical lymph node dissection offers the only chance of cure in both sporadic and hereditary syndromes. Careful follow-up in the postoperative period, with serial calcitonin levels at 6 months and then annually, is important to detect recurrent or persistent disease.

Identification and appropriate surgical treatment of hereditary MTC syndromes at an early age result in an excellent long-term prognosis. Prophylactic thyroidectomy in children can be performed with minimal morbidity and up to a $96 \%$ biochemical cure. ${ }^{60,61}$ Early diagnosis of FMTC, MEN 2A, and MEN 2B with RET screening and prophylactic thyroidectomy timed according to the risk level of individual RET mutations may allow treatment before MTC develops.

\section{References}

1. Gagel RF, Marx SJ. Multiple endocrine neoplasia. In: Larsen PR, Kronenberg HM, Melmed S, et al., eds. Williams Textbook of Endocrinology, 10th ed. Philadelphia: Saunders; 2002:1717-1762.

2. Takahashi M, Ritz J, Cooper GM. Activation of a novel human transforming gene, ret, by DNA rearrangement. Cell 1985;42: 581-588.

3. Mulligan LM, Kwok JB, Healey CS, et al. Germline mutations of the RET proto-oncogene in multiple endocrine neoplasia type 2A. Nature 1993;363:458-460.

4. Donis-Keller H, Dou S, Chi D, et al. Mutations of the RET protooncogene are associated with MEN 2A and FMTC. Hum Mol Genet 1993;2:851-856.

5. Eng C, Smith DP, Mulligan LM, et al. Point mutation within the tyrosine kinase domain of the RET proto-oncogene in multiple endocrine neoplasia type $2 \mathrm{~B}$ and related sporadic tumors. Hum Mol Genet 1994;3:237-241.

6. Saad MF, Ordonez NG, Rashid RK, et al. Medullary carcinoma of the thyroid. Medicine 1984;63:319-342.

7. Scollo C, Baudin E, Travagli JP, et al. Rationale for central and bilateral lymph node dissection in sporadic and hereditary medullary thyroid cancer. J Clin Endocrinol Metab 2003;88:2070-2075.

8. Quayle FJ, Moley JF. Medullary thyroid carcinoma: including MEN 2A and MEN 2B syndromes. J Surg Oncol 2005;89:122-129.

9. Reiff E, Kebebew E. Surgical treatment of medullary thyroid cancer.

10. Evans DB, Lee JE, Merrel RC, et al. Adrenal medullary disease in multiple endocrine Neoplasia type 2: appropriate management. Endocrinol Metab Clin North Am 1994;23:167-176.

11. Cohen R, Campos JM, Salaun C, et al. Preoperative calcitonin levels are predictive of tumor size and postoperative calcitonin 
Ogilvie and Kebebew

normalization in medullary thyroid carcinoma. J Clin Endocrinol Metab 2000;85:919-922.

12. Libbey NP, Nowakowski KJ, Tucci JR. C-cell hyperplasia of the thyroid in a patient with goitrous hypothyroidism and Hashimoto's thyroiditis. Am J Surg Pathol 1989;13:71-77.

13. Raue F, Frank-Raue K, Grauer A. Multiple endocrine neoplasia type 2: clinical features and screening. Endocrinol Metab Clin North Am 1994;23:137-156.

14. Pomares FJ, Canas R, Rodriguez JM, et al. Differences between sporadic and multiple endocrine Neoplasia type 2A phaeochromocytoma. Clin Endocrinol 1998;48:195-200.

15. Eng C, Mulligan LM, Smith DP, et al. Low frequency of germline mutations in the RET proto-oncogene in patients with apparently sporadic medullary thyroid carcinoma. Clin Endocrinol 1995;43: 123-127.

16. Wohllk N, Cote GJ, Bugalho MMJ, et al. Relevance of RET protooncogene mutations in sporadic medullary thyroid carcinoma. J Clin Endocrinol Metab 1996;81:3740-3745.

17. Yen TW, Shapiro SE, Gagel RF, et al. Medullary thyroid carcinoma: results of a standardized surgical approach in a contemporary series of 80 consecutive patients. Surgery 2003;134:890-899.

18. Machens A, Holzhausen HJ, Thanh PN, Dralle H. Enhancement of lymph node metastasis of thyroid carcinoma. Cancer 2003;98: 712-719.

19. Scollo C, Baudin E, Travagli JP, et al. Rationale for central and bilateral lymph node dissection in sporadic and hereditary medullary thyroid cancer. J Clin Endocrinol Metab 2003;88:2070-2075.

20. Callender DL, Sherman SI, Gagel RF, et al. Cancer of the thyroid. In: Myers EN, Suen JY, eds. Cancer of the Head and Neck. Philadelphia: WB Saunders;1996:485.

21. Kebebew E, Greenspan FS, Clark OH, et al. Extent of disease and practice patterns for medullary thyroid cancer. J Am Coll Surg 2005;200:890-896.

22. Kaltsas G, Rockall A, Papadogias D, et al. Recent advances in radiological and radionuclide imaging and therapy of neuroendocrine tumors. Eur J Endocrinol 2004;151:15-27.

23. McDougall IR, Davidson J, Segall GM. Positron emission tomography of the thyroid, with an emphasis on thyroid cancer. Nucl Med Commun 2001;22:485-492.

24. Abdelmoumene N, Schlumberger M, Gardet P, et al. Selective venous sampling catheterization for localization of persisting medullary thyroid carcinoma. Br J Cancer 1994;69:1141-1144.

25. Gilliland FD, Hunt WC, Morris DM, et al. Prognostic factors for thyroid carcinoma. A population-based study of 15,698 cases from the surveillance, epidemiology and end results (SEER) program 1973-1991. Cancer 1997;79:564-573.

26. Hundahl SA, Fleming ID, Fremgen AM, et al. A national cancer data base report on 53,856 cases of thyroid carcinoma treated in the U.S., 1985-1995. Cancer 1998;83:2638-2648.

27. Kebebew E, Ituarte PHJ, Siperstein AE, et al. Medullary thyroid cancer: clinical characteristics, treatment, prognostic factors and a comparison of staging systems. Cancer 2000;88:1139-1148.

28. Lippman SM, Mendelsohn G, Trump DL, et al. The prognostic and biologic significance of cellular heterogeneity in medullary thyroid carcinoma: a study of calcitonin, L-dopa decarboxylase, and histaminase. J Clin endocrinol Metab 1982;54:233-240.

29. Mendelsohn G, Wells SA Jr, Baylin SB. Relationship of tissue carcinoembryonic antigen and calcitonin to tumor virulence in medullary thyroid carcinoma. An immunohistochemical study in early, localized, and virulent disseminated stages of disease. Cancer 1984;54:657-662.
30. Faggiano A, Talbot M, Lacroix L, et al. Differential expression of galectin-3 in medullary thyroid carcinoma and C-cell hyperplasia. Clin Endocrinol (Oxf) 2002;57:813-819.

31. Van Heerden JA, Grant CS, Gharib H, et al. Long-term course of patients with persistent hypercalcitoninemia after apparent curative primary surgery for medullary thyroid carcinoma. Ann Surg 1990;212:395-400.

32. Porter AT, Ostrowski MJ. Medullary carcinoma of the thyroid treated by low-dose adriamycin. Br J Clin Pract 1990;44:517-518.

33. Shimaoka K, Shoenfeld DA, DeWys WD, et al. A randomized trial of doxorubicin versus doxorubicin plus cisplatin in patients with advanced thyroid carcinoma. Cancer 1985;56:2155-2160.

34. Schlumberger M, Abdelmoumene N, Delisle MJ, et al. Treatment of advanced medullary thyroid cancer with an alternating combination of 5 FU streptozocin and 5 FU-dacarbazine. Br J Cancer 1995;71: 363-365.

35. Wu LT, Averbuch SD, Ball DW, et al. Treatment of advanced medullary thyroid carcinoma with a combination of cyclophosphamide, vincristine and dacarbazine. Cancer 1994;73:432-436.

36. Nocera M, Baudin E, Pellegriti G, et al. Treatment of advanced medullary thyroid carcinoma with an alternating combination of doxorubicin-streptozocin and 5 FU-dacarbazine. Br J Cancer 2000; 83:715-718.

37. Petursson SR. Metastatic medullary thyroid carcinoma. Complete response to combination chemotherapy with dacarbazine and 5-fluorouracil. Cancer 1988;62:1899-1903.

38. Orlandi F, Caraci P, Berruti A, et al. Chemotherapy with dacarbazine and 5-fluorouracil in advanced medullary thyroid cancer. Ann Oncol 1994;5:763-765.

39. $\overline{\text { Di Bartolomeo M }}$, Bajetta E, Bohcicchio AM, et al. A phase II trial of dacarbazine, fluorouracil, and epirubicin in patients with neuroendocrine tumors. A study by the Italian Trials in Medical Oncology (I.T.M.O.) Ann Oncol 1995;6:77-79.

40. Nelkin BD, Ball DW. Combrestatin A-4 and doxorubicin combination treatment is effective in a preclinical model of human medullary thyroid carcinoma. Oncol Rep 2001;8:157-160.

41. Lupoli $G$, Cascone $E$, Arlotta F, et al. Treatment of advanced medullary thyroid carcinoma with a combination of recombinant interferon alpha-2b and Octreotide. Cancer 1996;78:1114-1118

42. Vitale $G$, Taliaferri P, Caraglia M, et al. Slow release lanreotide in combination with interferon alpha-2b in the treatment of symptomatic advanced medullary thyroid carcinoma. J Clin Endocrinol Metab 2000;85:983-988.

43. Cuccuru G, Lanzi C, Cassinelli G, et al. Cellular effects and antitumor activity of RET inhibitor RPI-1 on MEN 2A-associated medullary thyroid cancinoma. J Natl Cancer Inst 2004;96:1006-1114.

44. Cohen MS, Hussain HB, Moley JF. Inhibition of medullary thyroid carcinoma cell proliferation and RET phosphorylation by tyrosine kinase inhibitors. Surgery 2002;132:960-966.

45. Carlomagno F, Vitagliano D, Guida T, et al. ZD 6474, an orally available inhibitor of KDR tyrosine kinase activity, efficiently blocks oncogenic RET kinases. Cancer Res 2002;62:7284-7290.

46. Kodama $Y$, Asai N, Kawai K, et al. The RET proto-oncogene: a molecular therapeutic target in thyroid cancer. Cancer Sci 2005;96: 143-148.

47. Drosten M, Stiewe T, Putzer BM. Antitumor capacity of a dominant negative RET proto-oncogene mutant in a medullary thyroid carcinoma model. Hum Gene Ther 2003;14:971-982.

48. Juweid ME, Hajjar G, Stein R, et al. Initial experience with high dose radioimmunotherapy of metastatic medullary thyroid cancer using $\left.{ }^{131} \mathrm{I}-\mathrm{MN}-14 \mathrm{~F} 9 \alpha \mathrm{b}\right)_{2}$ anti-carcinoembryonic antigen $\mathrm{Mab}$ and AHSCR. J Nucl Med 2000;41:93-103. 
Thyroid Surgery for Medullary Thyroid Cancer Syndromes

49. Schott M, Feldkamp J, Klucken M, et al. Calcitonin-specific antitumor immunity in medullary thyroid carcinoma following dendritic cell vaccination. Cancer Immunol Immunother 2002;51: 663-668.

50. Samaan NA, Schultz PN, Hickey RC. Medullary thyroid carcinoma: prognosis of familial versus sporadic disease and the role of radiotherapy. J Clin Endocrinol Metab 1988;67:801-805.

51. Brierley J, Tsang R, Simpson WJ, et al. Medullary thyroid cancer: analyses of survival and prognostic factors and the role of radiation therapy in local control. Thyroid 1996;6:305-310.

52. Harmer C, Bidmead M, Shepard S, et al. Radiotherapy planning techniques for thyroid cancer. Br J Radiol 1998;71:1069-1075.

53. Brandi ML, Gagel RF, Angeli A, et al. Consensus guidelines for diagnosis and therapy of MEN type 1 and type 2. J Clin Endocrinol Metab 2001;86:5658-5671.

54. Donovan DT, Levy ML, Furst EJ, et al. Familial Cutaneous lichen amyloidosis in association with multiple endocrine Neoplasia type 2A: a new variant. Henry Ford Hosp Med J 1989;37:147-150.

55. Herfarth KK, Bartsch D, Doherty GM, et al. Surgical management of hyperparathyroidism in patients with multiple endocrine neoplasia type 2A. Surgery 1996;120:966-973.
56. Decker RA, Geiger JD, Cox CE, et al. Prophylactic surgery for multiple endocrine neoplasia type Iia after genetic diagnosis; is parathyroid transplantation indicated? World J Surg 1996;20: 814-820.

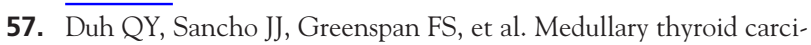
noma: the need for early diagnosis and total thyroidectomy. Arch Surg 1989;124:1206-1210.

58. Skinner MA, DeBenedetti MK, Moley JF, et al. Medullary thyroid carcinoma in children with multiplel endocrine Neoplasia types 2A and 2B. J Pediatr Surg 1996;31:177-181.

59. Eng C, Clayton D, Schuffenecker I, et al. The relationship between specific RET proto-oncogene mutations and disease phenotype in multiple endocrine neoplasia type 2. J Am Med Assoc 1996;276: 1575-1579.

60. Frilling A, Weber F, Tecklenborg C, et al. Prophylactic thyroidectomy in multiple endocrine neoplasia: the impact of molecular mechanisms of RET proto-oncogene. Arch Surg 2003;388:17-26.

61. Dralle H, Gimm O, Simon D, et al. Prophylactic thyroidectomy in 75 children and adolescents with hereditary medullary thyroid carcinoma; German and Austrian experience. World J Surg 1998;22: 744-751. 